\section{Quantification of human angiogenesis in immunodeficient mice using a photon counting-based method}

\author{
Zhihong Dong ${ }^{1}$, Kathleen G. Neiva ${ }^{1}$, Taocong Jin ${ }^{1}$, Zhaocheng Zhang1, \\ Daniel E. Hall ${ }^{1}$, David J. Mooney², Peter J. Polverini ${ }^{1}$, and Jacques E. Nör ${ }^{1}$ \\ 1 University of Michigan, Ann Arbor, MI and ${ }^{2}$ Harvard University, Cambridge, MA, USA
}

BioTechniques 43:73-77 (July 2007)

doi $10.2144 / 000112457$

Testing new antiangiogenic drugs for cancer treatment requires the use of animal models, since stromal cells and extracellular matrices mediate signals to endothelial cells that cannot be fully reproduced in vitro. Most methods used for analysis of antiangiogenic drugs in vivo utilized histologic examination of tissue specimens, which often requires large sample sizes to obtain reliable quantitative data. Furthermore, these assays rely on the analysis of murine vasculature that may not be correlated with the responses of human endothelial cells. Here, we engineered human blood vessels in immunodeficient mice with human endothelial cells expressing luciferase, demonstrated that these cells line functional blood vessels, and quantified angiogenesis over time using a photon counting-based method. In a proof-of-principle experiment with PTK/ZK, a small molecule inhibitor of vascular endothelial growth factor (VEGF) tyrosine kinase receptors, a strong correlation was observed between the decrease in bioluminescence (9.12-fold) in treated mice and the actual decrease in microvessel density (9.16-fold) measured after retrieval of the scaffolds and immunohistochemical staining of endothelial cells. The method described here allows for quantitative and noninvasive investigation into the effects of anti-cancer drugs on human angiogenesis in a murine host.

\section{INTRODUCTION}

Angiogenesis plays a key role in development and pathogenesis of several diseases (1). In vivo assays are essential for the understanding of mechanisms underlying the physiology and pathology of angiogenesis, as well as the discovery and evaluation of new therapeutic strategies (2). Most methods for the in vivo study of angiogenesis available today rely on the histological analyses of tissues retrieved from the animals. These assays typically do not allow for the continuous evaluation of changes over time in the same animal. Instead, they provide crossectional data, increasing the number of animals, and consequently, the cost and time for data gathering and processing.

A tissue engineering-based approach has been developed to generate human blood vessels in immunodeficient mice (3). Primary human dermal microvascular endothelial cells (HDMEC) seeded in biodegradable scaffolds and transplanted into severe combined immunodeficient (SCID) mice organize into functional human blood vessels that anastomize (connect) with the mouse vasculature and transport mouse blood (3). This model allows for the study of the responses of human blood vessels to antiangiogenic drugs. However, a major limitation of the current model is that it does not allow for the evaluation of responses over time in the same animal. Therefore, a typical experiment to determine response to a novel antiangiogenic drug requires large numbers of animals and tissue samples (4).

Here, we describe a new method based on photon counting that can be used to overcome this shortcoming. Photon counting allows for the quantification of cells expressing luciferase proteins with a unique signal-to-noise ratio and sensitivity (5-9). Most of the work described until now with photon counting has been performed in established cell lines or tumor cells. Using this new method we were able primary human endothelial cells; (ii) to (i) transduce the luciferase gene into demonstrate that these cells retained the ability to form functional blood vessels; and (iii) demonstrate the robustness of the model by using it to quantify the antiangiogenic effect of a well-established inhibitor of vascular endothelial growth factor (VEGF) signaling (PTK/ZK).

\section{MATERIALS AND METHODS}

\section{Primary Human Endothelial Cells Stably Expressing Firefly Luciferase}

To generate the luciferase expression construct pFB-neo-luc, the 1650-bp firefly luciferase gene was cloned into a retroviral vector containing the neomycin resistance cassette (pFB-Neo; Stratagene, La Jolla, CA, USA). Ecotropic packaging cells PE501 (gift of A.D. Miller) were transfected with either pFB-neo-luc or the empty vector pFB-Neo using Lipofectin ${ }^{\circledR}$ (Invitrogen, Carlsbad, CA, USA) following the manufacturer's instructions. The virus-containing supernatants of transfected PE501 cells were centrifuged at $1811 \times g$ for 10 min and mixed at 1:5 dilution in Dulbecco's modified Eagle's medium (DMEM) $/ 10 \%$ fetal bovine serum (FBS) to infect the amphotropic retroviral packaging cells PA317 (gift of A.D. Miller) as described (10). After 2 weeks' selection with $400 \mu \mathrm{g} / \mathrm{mL}$ G418 (Fisher Scientific, Fair Lawn, NJ, USA), virus-containing supernatant from PA317-luc or controls were centrifuged and diluted at 1:5 in endothelial cell growth medium (EGM2-MV; Cambrex, Walkersville, MD, USA) to infect primary human dermal microvascular endothelial cells (HDMEC; Cambrex) in the presence of $4 \mu \mathrm{g} / \mathrm{mL}$ polybrene (Sigma-Aldrich, St. Louis, MO, USA). The transduced endothelial cells [HDMEC expressing luciferase (HDMEC-luc) and empty vector control cells (HDMEC-neo)] were selected for at least 2 weeks with $250 \mu \mathrm{g} / \mathrm{mL}$ G418. The positive control squamous cell carcinoma cell line UM-SCC-17B-luc (17B-luc) and negative control cells UM-SCC-17neo (17B-neo) were generated previously in our laboratory (11). 


\section{Western Blot Analysis and Luciferase Activity Assay}

To confirm the expression of luciferase, cell lysates of HDMEC-luc, HDMEC-neo, 17B-luc, and 17B-neo were prepared as described previously (11). Proteins were resolved in a $10 \%$ Tris-glycine sodium dodecyl sulfate (SDS)-polyacrylamide gel, transferred to a nitrocellulose membrane (Bio-Rad Laboratories, Hercules, CA, USA), and exposed to monoclonal anti-luciferase antibody $(2 \mu \mathrm{g} / \mathrm{mL}$; Sigma-Aldrich) overnight at $4^{\circ} \mathrm{C}$. After incubation with horseradish peroxidase (HRP)-conjugated anti-mouse immunoglobulin $\mathrm{G}$ (IgG; Jackson ImmunoResearch, West Grove, PA, USA), membranes were exposed to an enhanced chemiluminescent substrate for detection of HRP (Pierce, Rockford, IL, USA). For luciferase activity assay, cell lysates were prepared with Passive Lysis Buffer (Promega, Madison, WI, USA) according to the manufacturer's instructions. Each cell lysate $(20 \mu \mathrm{L})$ was transferred to a 96-well luminometer plate containing $100 \mu \mathrm{L}$ Luciferase Assay Reagent II (Promega). Luciferase activity was measured using an LMax II luminometer (Molecular Devices, Sunnyvale, CA, USA).

\section{Severe Combined Immunodeficient Mouse Model of Human Angiogenesis}

The SCID mouse model of human angiogenesis was established and applied extensively (3,4,11-14). Briefly, $1 \times 10^{6}$ HDMEC-luc or HDMEC-neo or $1 \times 10^{5}$ 17B-luc were resuspended in a 1:1 mix of culture medium and Matrigel ${ }^{\mathrm{TM}}$ (BD Biosciences, San Diego, CA, USA) and seeded into a $6 \times 6 \times 1 \mathrm{~mm}$ biodegradable, highly porous poly-L-lactic acid scaffold (PLLA; Boehringer Ingelheim, Ingelheim, Germany). After a $30-\mathrm{min}$ incubation at $37^{\circ} \mathrm{C}$ in $5 \% \mathrm{CO}_{2}$, the scaffolds were implanted subcutaneously into the dorsum of SCID mice (CB-17; Charles River Laboratories, Wilmington, MA, USA). In the treatment group, the VEGF receptor tyrosine kinase inhibitor PTK/ ZK (PTK787/ZK222584; Novartis, Basel, Switzerland) in PEG300 (Fluka, Seelze, Germany) was orally administrated by gavage once a day at a dose of $50 \mathrm{mg} / \mathrm{kg}$ body weight starting 1 day after scaffold implantation, whereas mice in the control group received vehicle alone. At the time indicated, the implants were retrieved and fixed in $10 \%$ neutral buffered formalin overnight at $4{ }^{\circ} \mathrm{C}$ and processed for histology.

\section{Photon Counting-Based Method}

Image capture was performed on a cryogenically cooled IVIS ${ }^{\circledR}$ system coupled to the data acquisition software (Xenogen, Hopkinton, MA, USA) immediately after implantation and every 3-4 days thereafter for 21 days. Prior to imaging, mice were anesthetized with $2 \%$ inhaled isoflurane, and luciferin potassium salt (Xenogen) in PBS was administered intraperitoneally at a dose of $320 \mathrm{mg} / \mathrm{kg}$ body weight. Mice were placed on a thermostated bed in the IVIS chamber, and isoflurane was administered by a nose cone delivery system. Seventeen minutes after the luciferin injection, a grayscale body surface image was taken, followed by acquisition and overlay of the pseudocolor image representing the emission of the active bioluminescence in the implanted cells. Integration times of $30 \mathrm{~s}$ to $5 \mathrm{~min}$ were used for luminescent acquisition. Images were analyzed with Living Image ${ }^{\circledR}$ software (Xenogen), and the light intensity from the region of interest corresponding to the scaffold was measured in units of photons $/ \mathrm{s} / \mathrm{cm}^{2}$.
A

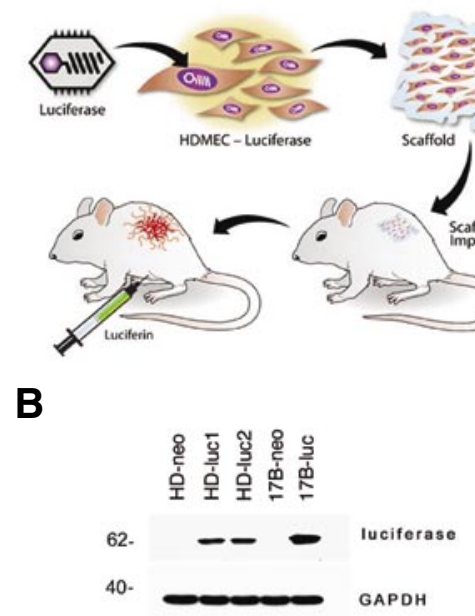

C

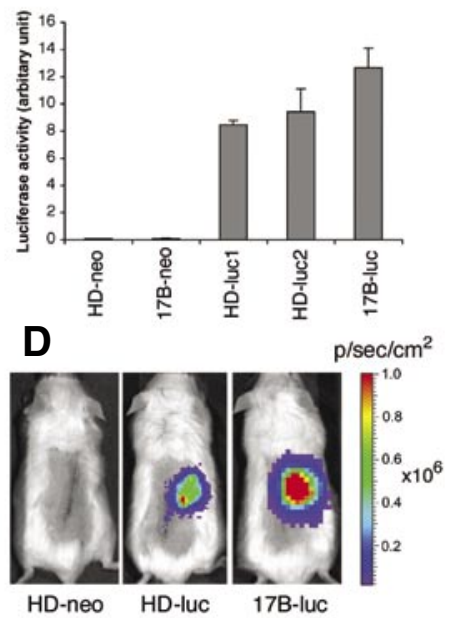

E

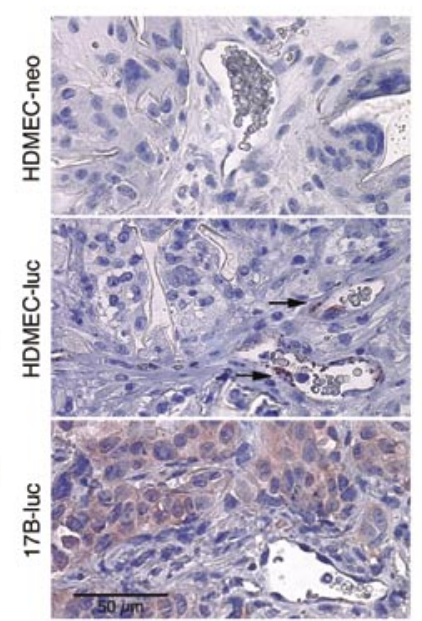

Figure 1. Characterization of murine model for quantification of human angiogenesis using a photon counting-based method. (A) Depiction of the photon counting-based method for quantification of human angiogenesis in murine hosts presented here. (B) Detection of luciferase expression by Western blot analysis with anti-luciferase antibody. (C) Quantification of luciferase expression by luciferase assays. (D) In vivo bioluminescence imaging of mice that received scaffolds seeded with human dermal microvascular endothelial cells (HDMEC) expressing luciferase (HDMEC-luc), empty vector control cells (HDMEC-neo), or UM-SCC-17B-luc (17B-luc). (E) Immunohistochemistry using anti-luciferase antibody depicting blood vessels lined with luciferase-positive blood vessel lining endothelial cells (arrows) in scaffolds seeded with HDMEC-luc, and luciferase-positive tumor cells in scaffolds seeded with 17B-luc $(400 \times)$. GAPDH, glyceraldehyde-3-phosphate dehydrogenase. 


\section{Immunohistochemistry}

After being deparaffinized in xylene, rehydrated, and washed in Tris-buffered saline (TBS), $\mathrm{pH} 8.0$, the histological sections were incubated in antigen retrieval solution (Dako, Carpinteria, CA, USA) for $20 \mathrm{~min}$ at $90^{\circ}-95^{\circ} \mathrm{C}$, followed by incubation with primary antibody at $4^{\circ} \mathrm{C}$ overnight. Rabbit antiluciferase antibody $(0.5 \mu \mathrm{g} / \mathrm{mL}$; Cortex, San Leandro, CA, USA) was used to identify cells (endothelial or tumor cells) expressing luciferase in scaffolds. Polyclonal anti-human factor VIII antibody (1:500 dilution; Lab Vision, Fremont, CA, USA) was used to stain the microvascular networks formed by the implanted human endothelial cells, as previously described (14). The number of microvessels in 10 random fields per implant was counted in three mice per condition under a light microscope at $200 \times$ magnification. Statistical significance throughout this study $(P \leq 0.05)$ was determined by Student's $t$-test or one-way analysis of variance (ANOVA), followed by the Student-Newman-Keuls test using the SigmaStat 2.0 software (SPSS, Chicago, IL, USA).

\section{RESULTS AND DISCUSSION}

The in vivo method for quantification of human angiogenesis presented here is conceptually based on the engineering of human blood vessels in immunodeficient mice using luciferase-transduced primary endothelial cells (Figure 1A). We transduced the luciferase construct into primary endothelial cells using a retroviral vector in vitro, and selected transduced cells with neomycin. Western blot analyses demonstrated that luciferase was highly expressed in HDMEC-luc and 17B-luc, compared with empty vector controls (Figure 1B). We confirmed the luciferase activity in the endothelial cells, and demonstrated that the activity levels in the HDMECluc were similar to the ones observed in 17B-luc cells (Figure 1C).

To evaluate if the proposed method based on photon counting allows for quantification of human angiogenesis, we seeded HDMEC-luc or HDMECneo into biodegradable scaffolds and

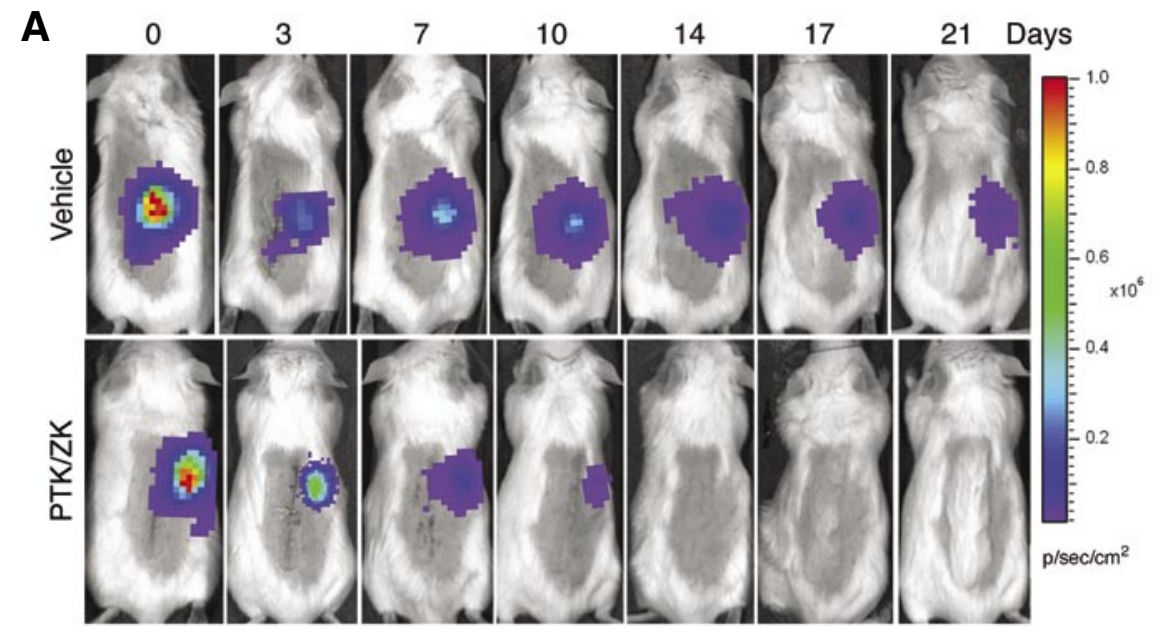

B
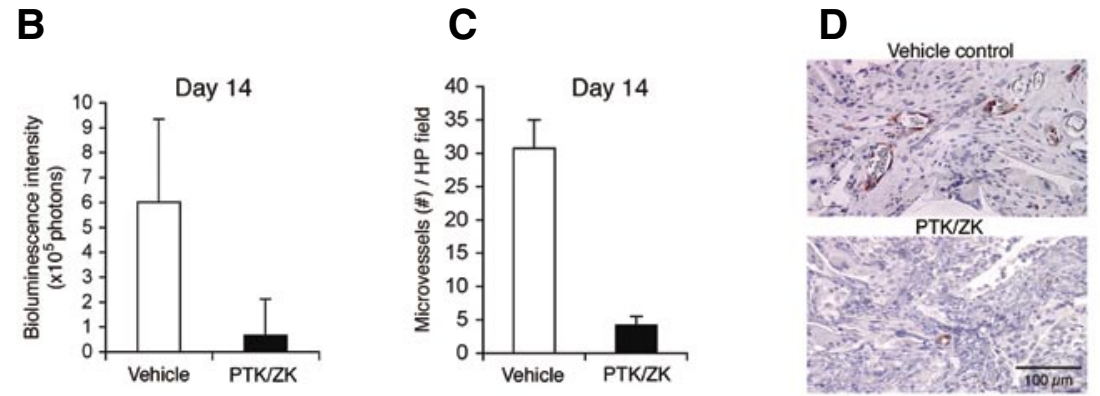

Figure 2. Proof-of-principle experiment for the validation of a photon counting-based method for angiogenesis investigation. (A) Images of representative mice implanted with scaffolds with human dermal microvascular endothelial cells (HDMECs) expressing luciferase (HDMEC-luc). Mice were treated with either vehicle (top row) or PTK/ZK (bottom row) for 21 days. (B) Graph depicting bioluminescence intensity 14 days after implantation in mice treated with either PTK/ZK or vehicle. (C) Graph depicting microvessel density 14 days after implantation in mice treated with either PTK/ZK or vehicle. Immunohistochemistry with factor VIII antibody was used identify microvessels. The number of microvessels in 10 random fields per scaffold was counted in at least three scaffolds/condition from independent mice under light microscopy $(200 \times)$. (D) Representative microscopic fields of tissue sections immunostained with factor VIII from mice that were either treated with PTK/ZK or vehicle $(400 \times)$.

transplanted into immunodeficient mice $(3,11)$. We observed strong bioluminescence in mice that received implants seeded with HDMEC-luc, whereas no bioluminescence was detected in mice with control implants containing HDMEC-neo (Figure 1D). To evaluate if the HDMEC-luc was lining the walls of the blood vessels inside the scaffolds, implants were retrieved and processed for histological examination. Luciferase expression was observed in the blood vessels of implants populated with HDMEC-luc, but not in control implants (Figure 1E). These data confirmed that HDMEC-luc organized into functional blood vessels, and that bioluminescence can be a readout for microvas- cular density. We performed control experiments in which scaffolds were seeded with 17B-luc. These scaffolds also presented strong bioluminescence (Figure 1D). However, in this case, the luciferase-positive cells (tumor cells) were dispersed throughout the scaffold and were absent from the blood vessel walls (Figure 1E). Taken together, these data demonstrate that this method provides the quantification of blood vessel-forming endothelial cells. The data also demonstrate the versatility of this method, since the same strategy can be used to measure tumor progression (using luciferase-positive tumor cells) or tumor angiogenesis (using luciferasepositive endothelial cells; Figure 1E). 
Next, we performed proof-ofprinciple experiments to determine the feasibility and sensitivity of this model for the evaluation of response to antiangiogenic treatment. PTK/ZK is an inhibitor of all known VEGFR tyrosine kinases with demonstrated antiangiogenic effect in animal models and phase I/II clinical trials (15-17). Starting the day after implantation of PLLA scaffolds (described in the Materials and Methods section) seeded with HDMEC-luc, mice received daily administration of PTK/ZK orally, and control mice received vehicle alone. In mice that did not receive PTK/ZK, we observed bioluminescence throughout the duration of the experiment (Figure 2A). In contrast, mice treated with PTK/ZK presented a rapid decrease in the intensity of the signal, which was minimal or absent by day 14 (Figure 2, A and B). To validate the assay and correlate the bioluminescence data with microvessel density, scaffolds were retrieved 14 days after implantation from an independent cohort of mice. Anti-human factor VIII antibody was used to immunolocalize the microvascular networks formed by the human endothelial cells within the scaffolds. We observed that a 9.12-fold decrease in bioluminescence (Figure 2B) correlated to a 9.16-fold decrease in microvessel density (Figure 2, C and D) in the PTK/ZK-treated group as compared with controls. The strong correlation between the fold-change in photon counts and microvessel density in response to treatment underscores the validity of the novel method presented here for in vivo angiogenesis studies. Notably, there was a significant decrease in bioluminescence immediately after implantation (i.e., from days $0-3$ ). We speculate that this is due to the fact that endothelial cells that are not capable of organizing themselves into functional blood vessels eventually die. This speculation is based on the observation that all the luciferase-positive endothelial cells identified by immunohistochemistry were found lining the walls of blood vessels (Figure 1E).

As with all experimental model systems, the proposed model has limitations. While fluorescence imaging is based on the actual visual- ization of blood vessels $(18,19)$, photon counting-based methods use a camera to capture bioluminescent data and superimpose pseudocolor images that represent the emission of the active bioluminescence of the implanted cells (20). Therefore, a photon counting-based method is quantitative and permits spatial and temporal analysis of the vasculature, but does not allow for direct visualization of blood vessels. Another limitation of the method is a decrease in bioluminescence observed in untreated control animals starting 21 days after implantation. One potential explanation for this decrease in bioluminescence could be the loss of the luciferase gene over time in primary endothelial cells. Nevertheless, in several independent experiments, we observed that the photon counts are constant for 17-21 days. Therefore, we believe this method to be ideally suited for investigations of the initial stages of angiogenesis.

In conclusion, we demonstrate that a photon counting-based method allows for noninvasive quantification of response to antiangiogenic treatment and is a convenient substitute for the more labor-intensive histological analysis of microvessel density. Compared with conventional methods, this model requires fewer animals, less time to evaluate data, and provides for a rapid, real-time, noninvasive way to study angiogenesis. Furthermore, this method allows for the quantitative analysis of the response of human (not mouse) functional blood vessels to novel antiangiogenic therapies, which enhances our capability of translating to the clinic the information gathered from laboratory experiments.

\section{ACKNOWLEDGMENTS}

We thank Chris Jung for his expertise in medical illustration, Tom Carey for providing us with the UM-SCC-17B cells, and Novartis/Schering AG for the PTK/ZK used in this study. Support for this research was provided by grants 1R01-DE14601, 1R01-DE15948, and 1R01-DE16586 from the NIH/NIDCR (to J.E.N.).

\section{COMPETING INTERESTS STATEMENT}

The authors declare no competing interests.

\section{REFERENCES}

1.Carmeliet, P. and P.K. Jain. 2000 Angiogenesis in cancer and other diseases. Nature 407:249-257.

2.Staton, C.A., S.M. Stribbling, S. Tazzyman, R. Hughes, N.J. Brown, and C.E. Lewis. 2004. Current methods for assaying angiogenesis in vitro and in vivo. Int. J. Exp. Pathol. 85:233-248.

3. Nör, J.E., M.C. Peters, J.B. Christensen, M.M. Sutorik, S. Linn, M.K. Khan, C.L. Addison, D.J. Mooney, and P.J. Polverini. 2001. Engineering and characterization of functional human microvessels in immunodeficient mice. Lab. Invest. 81:453-463.

4.Zeitlin, B.D., E. Joo, Z. Dong, K. Warner, G. Wang, Z. Nikolovska-Coleska, S. Wang, and J.E. Nör. 2006. Anti-angiogenic effect of TW37, a small molecule inhibitor of Bcl-2. Cancer Res. 66:8698-8706.

5. Contag, C.H. and B.D. Ross. 2002. It's not just about anatomy: in vivo bioluminescence imaging as an eyepiece into biology. J. Magn. Reson. Imaging 16:378-387.

6. Edinger, M., Y.A. Cao, Y.S. Hornig, D.E. Jenkins, M.R. Verneris, M.H. Bachmann, R.S. Negrin, and C.H. Contag. 2002. Advancing animal models of neoplasia through in vivo bioluminescence imaging. Eur. J. Cancer 38:2128-2136.

7. Kang, S.H., H.T. Cho, S. Devi, Z. Zhang, D. Escuin, Z. Liang, H. Mao, D.J. Brat, et al. 2006. Antitumor effect of 2-methoxyestradiol in a rat orthotopic brain tumor model. Cancer Res. 66:11991-11997.

8. Luo, J., A.H. Lin, E. Masliah, and T. WyssCoray. 2006. Bioluminescence imaging of Smad signaling in living mice shows correlation with excitotoxic neurodegeneration. Proc. Natl. Acad. Sci. USA 103:18326-18331.

9. Yanagihara, K., M. Takigahira, F. Takeshita, T. Komatsu, K. Nishio, F. Hasegawa, and T. Ochiya. 2006. A photon counting technique for quantitatively evaluating progression of peritoneal tumor dissemination. Cancer Res. 66:7532-7539.

10. Miller, A.D., D.G. Miller, J.V. Garcia, and C.M. Lynch. 1993. Use of retroviral vectors for gene transfer and expression. Met. Enzym. 217:581-599.

11. Pinsky, M.S., W. Song, Z. Dong, K. Warner, B. Zeitlin, E. Karl, D.E. Hall, and J.E. Nör. 2006. Activation of iCaspase-9 in neovessels inhibits oral tumor progression. J. Dent. Res. 85:436-441.

12. Nör, J.E., Y. Hu, W. Song, D.M. Spencer, and G. Nunez. 2002. Ablation of microvessels in vivo upon dimerization of iCaspase-9. Gene Ther. 9:444-451.

13.Song, W., Q. Sun, Z. Dong, D.M. Spencer, G. Nunez, and J.E. Nör. 2005. Anti-angiogenic gene therapy: disruption of neovascular networks mediated by inducible caspase- 9 


\section{Short Technical Reports}

delivered with a transcriptionally targeted adenoviral vector. Gene Ther. 12:320-329.

14. Nör, J.E., J.B. Christensen, D.J. Mooney, and P.J. Polverini. 1999. Vascular endothelial growth factor (VEGF)-mediated angiogenesis is associated with enhanced endothelial cell survival and induction of Bcl-2 expression. Am. J. Pathol. 154:375-384.

15.Lee, L., S. Sharma, B. Morgan, P. Allegrini, C. Schnell, J. Brueggen, R. Cozens, M. Horsfield, et al. 2006. Biomarkers for assessment of pharmacologic activity for a vascular endothelial growth factor (VEGF) receptor inhibitor, PTK787/ ZK 222584 (PTK/ZK): translation of biological activity in a mouse melanoma metastasis model to phase I studies in patients with advanced colorectal cancer with liver metastases. Cancer Chemother. Pharmacol. 57:761-771.

16. Wood, J.M., G. Bold, E. Buchdunger, R. Cozens, S. Ferrari, J. Frei, F. Hofmann, J. Mestan, et al. 2000. PTK787/ZK 222584, a novel and potent inhibitor of vascular endo- thelial growth factor receptor tyrosine kinases, impairs vascular endothelial growth factor-induced responses and tumor growth after oral administration. Cancer Res. 60:2178-2189.

17.Zakarija, A. and G. Soff. 2005. Update on angiogenesis inhibitors. Curr. Opin. Oncol. 17:578-583.

18. Yang, M., E. Baranov, X.M. Li, J.W. Wang, P. Jiang, L. Li, A.R. Moossa, S. Penman, and R.M. Hoffman. 2001. Whole body and intravital optical imaging of angiogenesis in orthotopically implanted tumors. Proc. Natl. Acad. Sci. USA 98:2616-2621.

19. Amoh, Y., M. Yang, L. Li, J. Reynoso, M. Bouvet, A.R. Moossa, K. Katsuoka, and R.M. Hoffman. 2005. Nestin-linked green fluorescent protein transgenic nude mouse for imaging human tumor angiogenesis. Cancer Res. 65:5352-5357.

20. Doyle, T.C., S.M. Burns, and C.H. Contag. 2004. In vivo bioluminescence imaging for integrated studies of infection. Cell. Microbiol. 6:303-317.
Received 10 November 2006; accepted 27 February 2007.

Address correspondence to Jacques Eduardo Nör, Angiogenesis Research Laboratory, University of Michigan, 1011 N. University, Rm. 2309, Ann Arbor, MI 48109-1078, USA. e-mail:jenor@umich.edu

To purchase reprints of this article, contact: Reprints@BioTechniques.com 